\title{
Assessment of $\beta$ Angle among the various facial Tupes in Garhwali Population - A Cephalometric Evaluation
}

\author{
Dr P Narayana Prasad,' Dr Rubina Ansari,, Dr Tarun Rana, ${ }^{3}$ Dr Nimme Rawat4 \\ 'Professor \& Head, 2.4Postgraduate student, 3Senior lecturer \\ Department of Orthodontics, Seema Dental College, Rishikesh, India
}

Correspondence: fiza_charmed@redifmail.com

\section{ABSTRACT}

Objective: The purpose of the study was to evaluate Beta $(\beta)$ angle variation in different facial types among Garhwali population and also to evaluate the differences in Beta angle in male and female subgroups.

Materials \& Method: The sample included pre-treatment lateral cephalograms of 90 native Garhwali subjects (age 16-24 years) who were divided into three groups: normodivergent, hypodivergent and hyperdivergent; of 30 subjects of each group 15 were male and 15 were female.

Result: In Garhwali population subjects with a $\beta$ angle between $28^{\circ}$ to $32^{\circ}$ have a normodivergent facial type (ANOVA $p<0.01$ ).

Conclusion: There is a significant difference in the mean values of $\beta$ angle in Garhwali population among various facial types.

Key words: Beta angle, hyperdivergent, hypodivergent, normodivergent

\section{Introduction}

Over the years diagnosis and treatment planning in orthodontics and dentofacial orthopaedics have relied substantially on technological and mechanical aids. These technological aids include imaging, articulators, jaw tracking and functional analyses. The goal in using these techniques is to accurately replicate or portray the "anatomic truth" to show the three dimensional anatomy in both static and dynamic function as it exists in nature. Imaging is one of the most ubiquitous tools orthodontists use to measure and record the size and form of craniofacial structures. Imaging has traditionally been used to record the status quo of limited or grouped anatomic structures.'

An accurate antero-posterior (AP) measurement of jaw relationships is critically important in orthodontic diagnosis and treatment planning. Both angular and linear measurements have been incorporated into various cephalometric analyses to help the clinician diagnose antero-posterior discrepancies and establish the most appropriate treatment plan. ${ }^{2,3,4}$ Any cephalometric analysis based on either angular or linear measurements has various shortcomings. Reliability of these parameters is questionable due to problems encountered in identification, reproducibility and effects of changes in occlusion and rotation of jaws during growth. ${ }^{5}$

A measurement was developed by Baik and Ververidou in the year 2004 named as the $\beta$ angle in an attempt to overcome the shortcomings of various other reference parameters used. ${ }^{5}$ This angle does not depend on any cranial landmarks or dental occlusion and reflects true antero-posterior changes as a result of growth and orthodontic intervention. Furthermore, it is not influenced by changes in occlusion thus it would be 
especially valuable whenever previously established cephalometric measurements such as ANB angle and Wits appraisal cannot be accurately used. ${ }^{5}$

Hence this study was undertaken with the aim and objective of evaluating the $\beta$ angle variations in Garhwali population.

\section{MATERIALS AND METHOD}

The present study was based on lateral cephalograms of 90 native Garhwali subjects who visited the outpatient department of Seema Dental College \& Hospital, Rishikesh, India for orthodontic alignment of teeth. The pre-treatment lateral cephalograms were obtained in the natural head position. According to their facial pattern the subjects were divided into three groups of 30 samples each comprising 15 males and 15 females using four parameters. The subjects were within the age range of 16-24 years, with the mean age of 18 years for female and 20 years for male.

The four parameters that were used to segregate the facial types into three groups were Jarabak ratio (Rakosi), mandibular plane angle (Steiner's), Y-axis of growth (Down's) and ALFH/AUFH (Di Paolo's).

The three groups were as follows: ${ }^{6}$

Group A- Normodivergent: The subjects in this group had normal mandibular plane angle, average anterior lower face height equal to the upper face height, normal $Y$-axis of growth, Jarabak ratio between $62 \%$ and $65 \%$.

Group B- Hyperdivergent: The subjects in this group had steep mandibular plane angle, long anterior lower face height, increased Y-axis of growth, Jarabak ratio $<62 \%$.

Group C- Hypodivergent: The subjects were grouped as hypodivergent on the basis of low mandibular plane angle and short lower facial height, decreased $Y$-axis of growth, Jarabak ratio $>65 \%$.

Each radiograph was scanned into an X-Y co-ordinate system using Epson perfection V700 photo scanner and was digitized by a single investigator using specific points required by the software. Linear and angular hard tissue measurements; skeletal and dental parameters were calculated electronically using the Dolphin Imaging ${ }^{\circledR} 11.0$ software (Dolphin Imaging and Management Solutions, Chatsworth, Calif.) and recorded in print.
Cephalometric points used were:

\begin{tabular}{|l|l|}
\hline Sella & Nasion \\
\hline Porion & Orbitale \\
\hline Pterygo maxillary fissure & Anterior Nasal Spine \\
\hline Posterior Nasal Spine & Point A \\
\hline J point & Centre of Condyle \\
\hline Ramus point & Articulare \\
\hline Point B & Pogonion \\
\hline Gnathion & Menton \\
\hline Gonion & \\
\hline
\end{tabular}

Reference planes used were:

- Sella-Nasion Plane (SN)

- $\quad$ Frankfort Horizontal Plane (FHP)

- Palatal Plane (PP)

- Mandibular Plane (Go-Gn) (Steiner's)

Various parameters used were (Figure 1):

- Mandibular plane angle - (Go - Gn to SN)

- $\quad$ Y axis of growth - (S - Gn to FH)

- $\quad$ alfh/AufH - N - Pt A (PP); Pt A on PP to Pt B on Go-Gn

- Jarabak ratio - S Go / N Me x 100

- $\quad B$ angle - pt. $A \perp C B-A B$

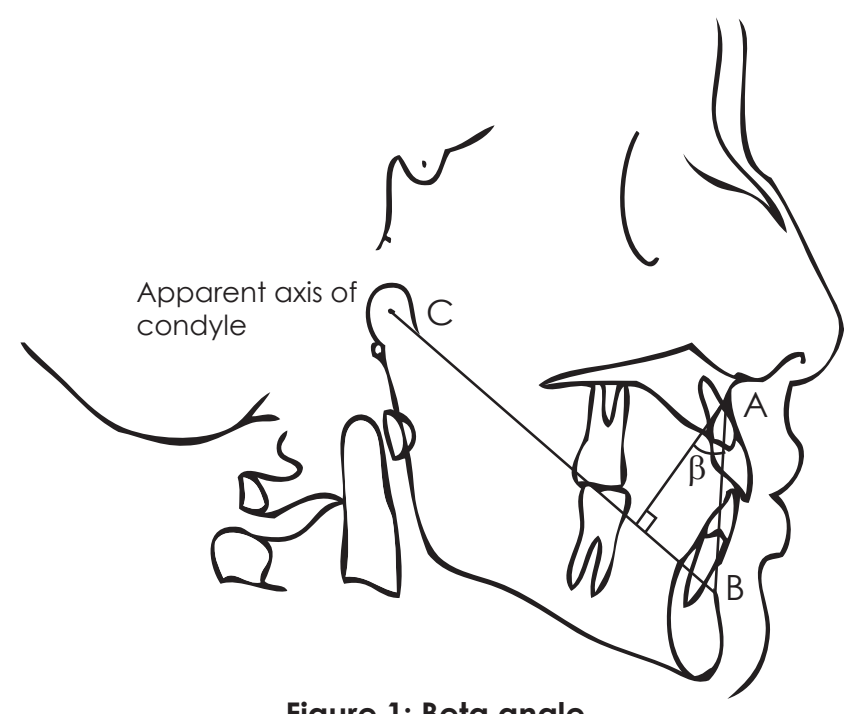

Figure 1: Beta angle

The data was summarized by finding mean and standard deviation. 1-way analysis of variance (ANOVA) was used followed by student t-test. A-P value $<0.01$ was considered to be statistically significant. 


\section{RESULTS}

Table 1 shows mean, standard deviation and variance of $\beta$ angle among three groups for males. There is highly significant difference between the measurements of $\beta$ angle among the males of these three groups ( $p<0.01)$.

Table 2 shows mean, standard deviation and variance of $\beta$ angle among three groups for females. There is highly significant difference between the measurements of $\beta$ angle among the males of these three groups ( $p<0.01)$.

Table 3 shows the comparison of mean $\beta$ angle value of the gender within the group, which shows non-significant difference.

Table 1: Descriptive statistics of $\beta$ angle and difference between the groups among male samples

\begin{tabular}{|l|c|c|c|c|}
\hline Groups & Mean & Standard deviation & Variance & p-value \\
\hline Normodivergent & 28.63 & 4.41 & 19.44 & $<0.01^{*}$ \\
\hline Hyperdivergent & 33.88 & 4.28 & 18.35 & $<0.01^{*}$ \\
\hline Hypodivergent & 24.26 & 4.72 & 22.32 & $<0.01^{*}$ \\
\hline
\end{tabular}

* Statistically significant

Table 2: Descriptive statistics of $\beta$ angle and difference between the groups among female samples

\begin{tabular}{|l|c|c|c|c|}
\hline Groups & Mean & Standard deviation & Variance & $p$-value \\
\hline Normodivergent & 30.69 & 2.87 & 8.27 & $<0.01^{*}$ \\
\hline Hyperdivergent & 32.29 & 4.28 & 18.32 & $<0.01^{*}$ \\
\hline Hypodivergent & 25.98 & 4.20 & 17.61 & $<0.01^{*}$ \\
\hline
\end{tabular}

* Statistically significant

Table 3: Comparison of $\beta$ angle between gender in each group

\begin{tabular}{|l|c|c|c|}
\hline Groups & Mean & Standard deviation & p-value \\
\hline Normodivergent & 28.63 & 30.69 (NS) & 0.14 \\
\hline Hyperdivergent & 33.88 & 32.29 (NS) & 0.31 \\
\hline Hypodivergent & 24.26 & 25.98 (NS) & 0.3 \\
\hline
\end{tabular}

NS Not significant

\section{DISCUSSION}

An accurate antero-posterior measurement of jaw relationship is critically important in orthodontic treatment planning. ${ }^{5}$ Both angular and linear variables have been proposed to analyze sagittal jaw relationship and jaw position. However the angular measurements are erroneous as changes in facial height and jaw inclination could lead to an alteration in these values and the linear measurements can be affected by the inclination of the reference line. ${ }^{7.8}$ The most popular parameter ANB angle can also be affected by various factors thus it is often misleading. The other alternative is Wits appraisal but it has a problem in encountering the functional occlusal plane. ${ }^{5}$

In the present study, $\beta$ angle was proposed as a stable measurement as all the reference points are located on the jaws and it is reliable to measure sagittal discrepancy. $\beta$ angle reflects true changes in the jaws and it can be used for consecutive comparisons. ${ }^{5}$ It is also useful for planning orthognathic surgeries as 
it helps to discriminate between patients of different facial types.

Thus the present study was undertaken to evaluate $\beta$ angle in Garhwali population and it was concluded that, for male samples in Group A (normodivergent) this angle was seen to be at an average of $28.63^{\circ}$; for female samples the $\beta$ angle was found to be at an average of $30.69^{\circ}$ for Group A (normodivergent) population.

In Group B (hyperdivergent) population, because of steep mandibular plane there is a downward and backward rotation of the mandible $e^{9,10}$ due to which there is an increase in $\beta$ angle. In Group $C$ (hypodivergent) population, because of the upward and anterior rotation of the mandible, there is a reduction in $\beta$ angle. ${ }^{11}$

\section{CONCLUSION}

According to the results of the cephalometric study on $\beta$ angle among the various facial types of Garhwali population; following conclusions can be drawn:

1. Previously established measurements for assessing sagittal jaw relationship can often be inaccurate.

2. A new angle; the Beta angle was developed as a diagnostic aid to evaluate the sagittal jaw relationship more consistently.

3. Garhwali population with a $\beta$ angle between $28^{\circ}$ and $32^{\circ}$ have normodivergent facial pattern; $\beta$ angle less than $28^{\circ}$ indicates hypodivergent facial pattern, and $\beta$ angle greater than $32^{\circ}$ indicates hyperdivergent facial pattern.

4. There is no statistically significant difference between mean $\beta$ angle values between male and female samples.

\section{OJN}

\section{REFERENCES}

1. Quintero JC, Trosien A. Craniofacial imaging in orthodontics: historical perspective, current status, and further developments. Angle Orthod 1999; 69(6):491-3.

2. Moyers RE, Bookstein FL, Guire KE. The concept of pattern in craniofacial growth. Am J Orthod 1979; 76:136-148.

3. Steiner: Cephalometrics in clinical practice. Angle Orthod 1959: 29: 8-29.

4. Chang HP. Assessment of antero-posterior jaw relationship. Am J Orthod Dentof Orthop 1987; 92:1 17-122.

5. Baik CY, Ververidou M: A new approach of assessing sagittal discrepancies: the Beta angle. Am J Orthod Dentofac Orthop 2004; 126:100-105.

6. Rana T, Khanna R, TikkU T, Sachan K: Relationship of maxilla to cranial base in different facial type: A cephalometric evaluation. JIOBCR 2012; 2(1):30-35.

7. Sachdeva $\mathrm{K}$ et al. Comparison of different angular measurements to assess sagittal skeletal discrepancy: A cephalometric study. IJODS 2012; 4(2):27-30.

8. Nanda R, Merrill RM. Cephalometric assessment of sagittal relationship between maxilla and mandible: Am J Orthod Dentofac Orthop 1994; 105:328-44.

9. Sassouni V. Classification of skeletal facial types. Am J Orthod1969; 55:109-23.

10. Schendel SA, Bell WH, Epker. The long face syndrome: Vertical maxillary excess. Am J Orthod 1976; 70:398-408.

11. Opdebeeck H, Bell WH. The short face syndrome. Am J Orthod Dentof Orthop 1978; 73:499-511. 